\title{
The MUSE Experiment: Studying the Proton Radius Puzzle with muon-proton Elastic Scattering
}

\author{
Katherine Mesick ${ }^{* \dagger}$ \\ Rutgers University \\ E-mail: kamyersejlab.org
}

\begin{abstract}
The Proton Radius Puzzle refers to the disagreement between the proton charge radius determined from muonic hydrogen and the radius determined from atomic hydrogen level transitions and $e p$ elastic scattering form factor data. The discrepancy of $\approx 7 \sigma$ is not yet explained, and though numerous resolutions have been proposed there is no generally accepted resolution to the puzzle. The MUon Scattering Experiment (MUSE) Collaboration will simultaneously measure elastic ep and $\mu p$ scattering at the Paul Scherrer Institute, testing the interesting possibility that electrons and muons behave differently. The experiment will also measure scattering with both polarities of $e$ and $\mu$, directly disentangling two-photon exchange effects. We plan to measure in the kinematic region of $Q^{2}=0.002-0.07 \mathrm{GeV}^{2}$, and determine the relative cross sections to a few tenths of a percent. This will allow the proton radius difference to be extracted to $\sim 0.01 \mathrm{fm}$, similar to the significance of the current measurements of the discrepancy.
\end{abstract}

16th International Workshop on Neutrino Factories and Future Neutrino Beam Facilities 25 -30 August, 2014

University of Glasgow, United Kingdom

${ }^{*}$ Speaker.

${ }^{\dagger}$ for the MUSE Collaboration. 


\section{Introduction}

The proton radius puzzle has existed since 2010, when a precise measurement of the proton charge radius from the Lamb shift in muonic hydrogen [1] found with high significance a $4 \%$ smaller value than the expected value based on previous measurements using atomic hydrogen Lamb shift and electron scattering. The muonic result has been confirmed [2], and many theories (see review by Pohl et al. [3]) to explain the puzzle have been proposed.

The proton radius can be extracted from two independent methods for each lepton probe. The first is through lepton-proton scattering data, where the radius is given by the slope of the electric form factor at $Q^{2}=0$ :

$$
\left\langle r_{p}^{2}\right\rangle \equiv-\left.6 \hbar^{2} \frac{d G_{E}\left(Q^{2}\right)}{d Q^{2}}\right|_{Q^{2}=0}
$$

The second method measures the Lamb shift in hydrogen which is directly sensitive to the proton radius. For electronic measurements, these two methods agree and give a radius of $\approx 0.88 \mathrm{fm}$. However, the muonic hydrogen measurements yield a radius of $\approx 0.84 \mathrm{fm}$. Measurements of $\mu p$ scattering date back to the 1970s-80s $[9,10]$, and are not precise enough nor low enough in $Q^{2}$ to have a clear impact. A recent summary of proton radius extractions is shown in Fig. 1.

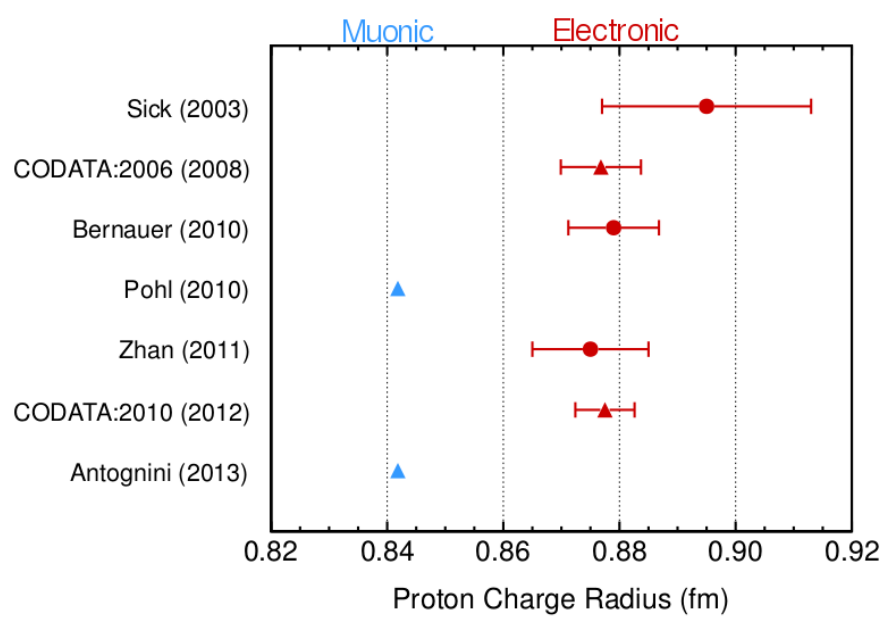

Figure 1: Results from recent extractions of the proton charge radius. The two precise muonic hydrogen measurements from Pohl et al. [1] and Antognini et al. [2] are on the left. The electronic measurements on the right include scattering measurement analyses from Sick [4], Bernauer et al. [5], Zhan et al. [6], and the global analysis of CODATA that includes atomic hydrogen [7, 8].

Possible resolutions include new physics beyond the Standard Model or new novel hadronic physics. In addition, the question of experimental issues has not been completely ruled out. To this regard, several experiments are planned or underway: pushing the ep scattering data to lower $Q^{2}$, redoing atomic hydrogen to higher precision, comparing the radius in heavier systematics $\left(\mathrm{D},{ }^{3} \mathrm{He}\right.$, ${ }^{4} \mathrm{He}$ ), and the subject of this paper, the MUSE experiment. MUSE will simultaneously measure $e^{ \pm} p$ and $\mu^{ \pm} p$ scattering for a direct comparison of the radius extracted for these two probes. This experiment will provide a missing piece of the proton radius puzzle, namely the proton charge radius extracted from $\mu p$ scattering with respect to that from $e p$ scattering. 


\section{MUSE Overview}

The MUon Scattering Experiment (MUSE) [11] will run at the Paul Scherrer Institute, where there is an intense separated $e, \mu, \pi$ beam. The cross section for $\mu p$ scattering will be measured to higher precision than the previous experiments, and to lower $Q^{2}$ for better sensitivity to the radius. MUSE will measure $e p$ and $\mu p$ scattering simultaneously, allowing for the cancellation of several systematic uncertainties and therefore a more precise comparison of the cross section, form factors, and extracted radius for the two lepton probes. Lastly, MUSE will employ both particle polarities to directly measure the contribution of two-photon exchange, looking for any enhanced effects that may lead to the observed discrepancy.

A cartoon of the experimental setup is shown in Fig. 2. Meeting the precision required by the

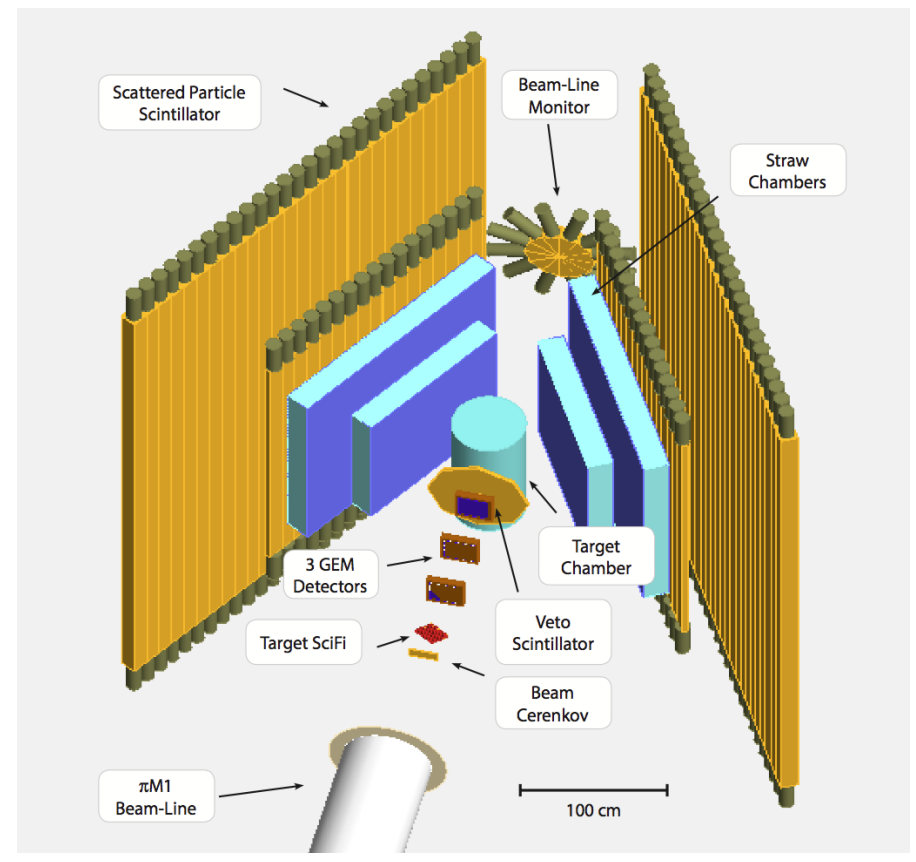

Figure 2: Geant 4 cartoon of the MUSE setup. The beam particles are tracked into an $\mathrm{LH}_{2}$ target by an array of scintillating fibers and a GEM telescope. The scattered particles are tracked by straw tube chambers and detected by high-precision scintillator walls. A beam monitor sits downstream of the target to monitor beam stability, and an annular veto scintillator sits upstream of the target to reject upstream decays.

experimental goals requires a large acceptance, precise particle timing, and good track resolution of the beam particles and the scattered particles. MUSE will have redundant scattered-particle detectors to the left and right of the beamline, covering an angular range of $\theta=20-100$ degrees and $\phi= \pm 45$ degrees. Two straw tube chambers on each side of the beamline will track the scattered particles with a position and angle resolution of $\approx 150 \mu \mathrm{m}$ and $1 \mathrm{mr}$, respectively. Two highprecision $(\approx 50 \mathrm{ps})$ scintillator walls will record energy deposited and precise timing information for the scattered particles.

Precise timing of the beam particles is required to achieve good particle identification (PID) based on the RF time of the particles in the experimental area. For the three momenta chosen for 
the experiment, $\approx 115,153$, and $210 \mathrm{MeV} / \mathrm{c}$, there is good separation in RF time for PID, as shown in Fig. 3. Timing at the $\approx 1 \mathrm{~ns}$ level will be used for the main beam PID in the trigger and will be provided by an array of scintillating fibers about $80 \mathrm{~cm}$ upstream of the target. At the analysis level, this timing resolution can be improved to $\approx 300$ ps. A beam Čerenkov, located about $90 \mathrm{~cm}$ upstream of the target, with a timing resolution of $\leq 100 \mathrm{ps}$ will be used in conjunction with the high-precision scintillator walls to provide a precise time-of-flight for helping distinguish muon decays from muon scattering events in the target (see Section 3).

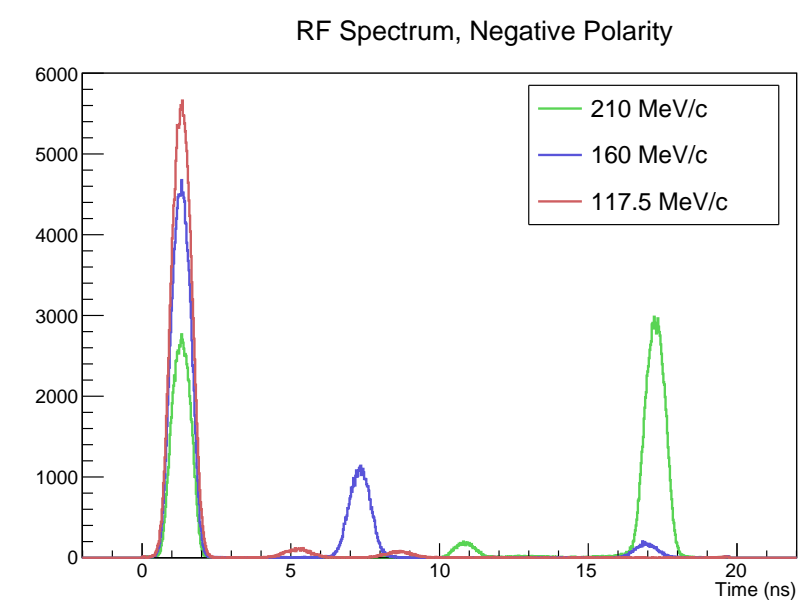

Figure 3: The RF time spectrum (time-of-flight) of particles at the location of the target as measured in beam tests in June 2014 showing good timing separation between the three peaks. The peaks from left to right correspond to $e, \mu$, and $\pi$ for $210 \mathrm{MeV} / \mathrm{c}$ and $e, \pi \mu$ for $160 \mathrm{MeV} / \mathrm{c}$ and $117.5 \mathrm{MeV} / \mathrm{c}$.

The remaining elements of the experimental setup include a GEM telescope to track beam particles into the target and determine the incident trajectory to $0.5 \mathrm{mr}$, a veto scintillator ring to veto most upstream decays and events that would hit the target chamber walls, and a beam monitor scintillator downstream of the target to monitor beam momentum stability and veto Møller scattering events.

The experiment will limit the beam rate to $5 \mathrm{MHz}$ using a collimator at the intermediate focus $\approx 12 \mathrm{~m}$ upstream of the target. Pions will be rejected at the trigger level with high efficiency through PID based on the RF time, resulting in an expected DAQ rate of $2-3 \mathrm{kHz}$. Triggering will be be handled by FPGAs onboard TRB3 readout boards, which also provide the precise timing information for the experiment. Standard QDCs will be used so that offline pulse-height corrections can be performed.

\section{Beam Tests and Simulations}

MUSE has conducted several test measurements at PSI between 2012 - present. These measurements have ranged from testing data acquisition hardware and software, measuring the beam particle fractions and spot size at the target, studying backgrounds in the beam, testing prototype scintillating fiber detectors and beam Čerenkovs, and performing a mini-scattering experiment. Figure 4 shows example results from the test measurements. 

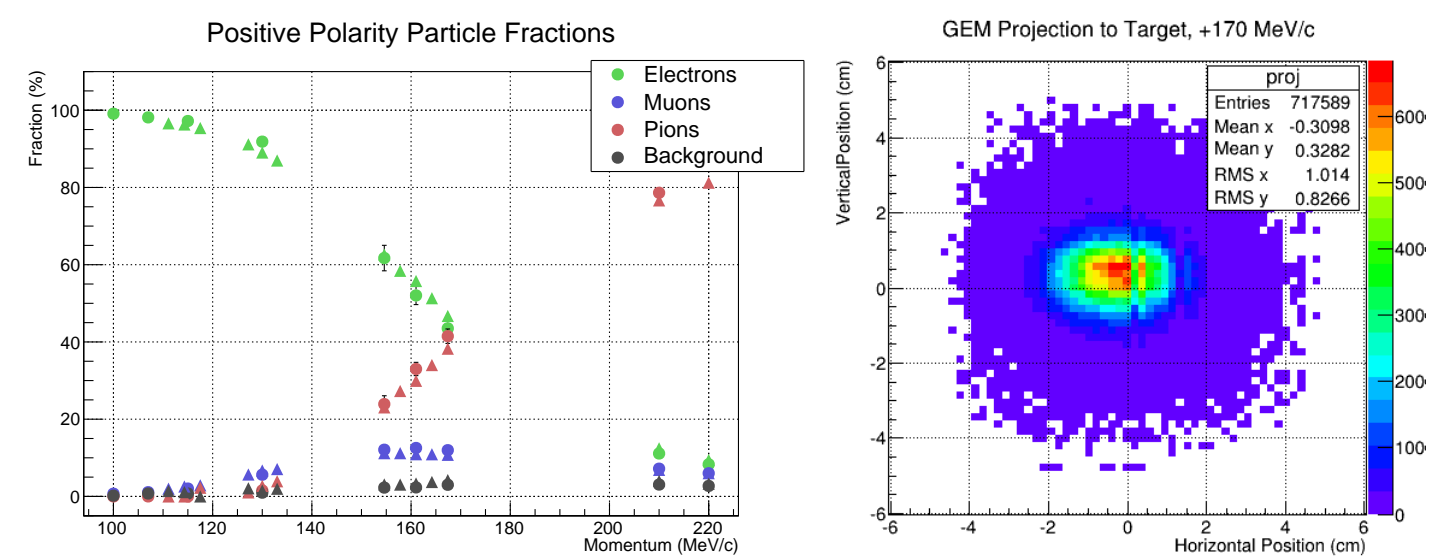

Figure 4: Examples from beam test measurements at PSI. Left: a measurement of the particle fractions versus beam momentum for positive polarity. Right: a measurement of the beam spot at the target using the GEM detectors, showing a $1 \mathrm{~cm} \times 0.8 \mathrm{~cm}$ beam spot. The spot size is independent of particle type.

Simulations of the experiment are underway using Geant4. In particular, the simulations are being used to optimize the design and positioning of the detectors and to study backgrounds. The main problematic backgrounds for the experiment are Møller scattering events from the target, and muons that decay in the target. Figure 5 shows an example of the latter, which demonstrates how this event is problematic due to a clean trigger and track reconstruction back to the target. The only way to separate these events from $\mu p$ scattering events in the target is through the time-of-flight from the beam Čerenkov and the scintillator walls. Due to the conversion of the muon to an electron in the target, the $\mu$-decay event will reach the scintillator walls a small amount of time sooner. This is demonstrated in the simulation on the right of Fig. 5.
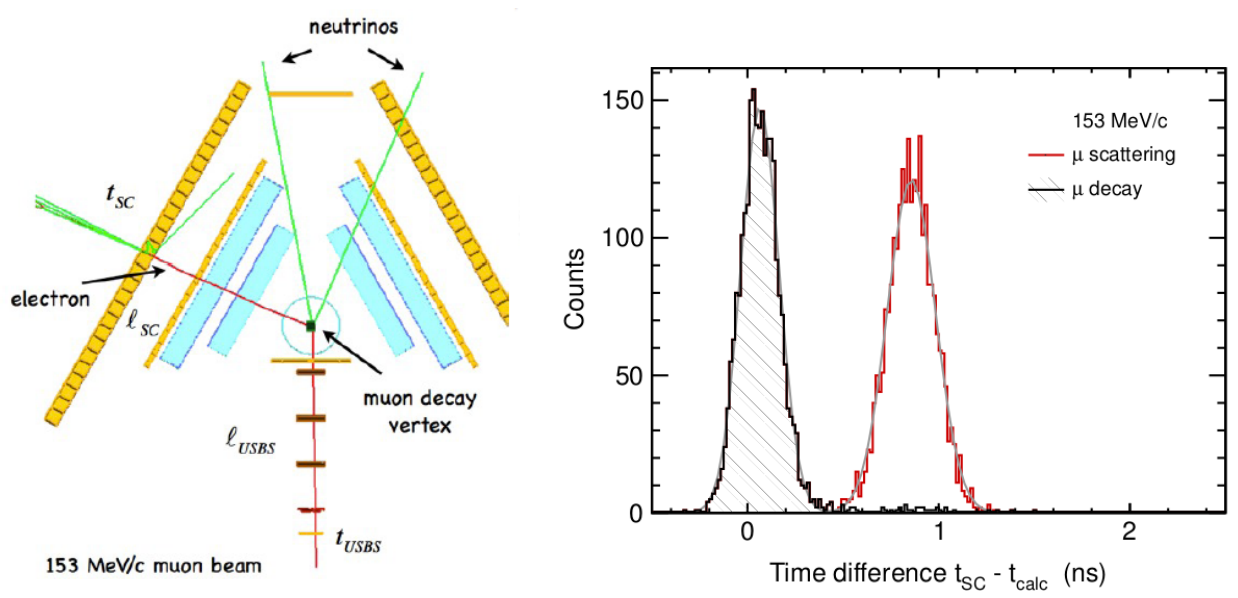

Figure 5: Example of muon decay simulations in Geant4. Left: View of a muon decaying in the target, where the electron is detected and tracks back to the target. Right: The time difference between the scintillator wall and the beam Čerenkov showing how the muon decay event can be rejected at the analysis level through this precise timing information. 


\section{Expected Results}

MUSE plans to take production data from 2017 - 2018. In the relative cross section comparison that MUSE plans, several systematic uncertainties such as the absolute target density and the efficiency of the beamline detectors drop out. The remaining uncertainties are related to point-topoint systematics, which MUSE will determine to $0.6 \%(0.4 \%)$ in the cross section for electrons (muons). The difference in uncertainty comes from the uncertainty in the radiative corrections, which are larger for electrons.

Figure 6 shows a conservative estimate of the uncertainties in the ratio of the extracted electric form factor $\left(G_{E}(\mu p) / G_{E}(e p)\right)$ for the three momentum settings. The uncertainty at forward angles is dominated by the point-to-point systematic error, while at large angles it is dominated the the uncertainty related to subtracting the $\mu$-decay background. An overall uncertainty of $0.1 \%$ related to removal of the magnetic term is not included. If the radius discrepancy of $4 \%$ is correct, then the difference in the slope of the form factor between muons and electrons will be seen at the $8 \%$ level.

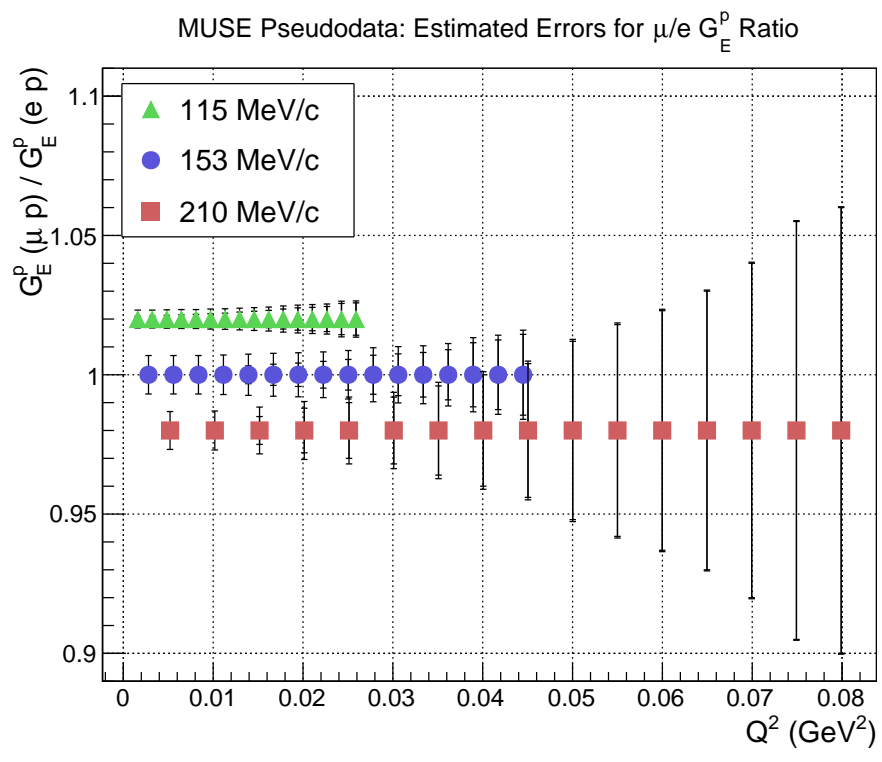

Figure 6: A conservative estimate of the uncertainty in the ratio $G_{E}(\mu p) / G_{E}(e p)$ from the MUSE measurement. There is a known (small) difference in the form factors due to a small difference in the effective $Q^{2}$ for electrons and muons, while this plot is normalized to 1 with offsets for plotting. A $4 \%$ difference in the charge radius correspond to an $8 \%$ difference in the slope of the form factors.

The radius will be extracted from the $e p$ and $\mu p$ scattering data, and by a relative comparison the expected sensitivity to the difference in the radius for the two probes is expected to be better than $0.01 \mathrm{fm}$. This gives an $\approx 4 \sigma$ measurement of the current discrepancy. This is demonstrated in Fig. 7 which shows the disagreement of the current extractions with the recent muonic hyodrogen result, and with the future MUSE point placed arbitrarily at $R_{p}-R_{\mu H}=0$. 


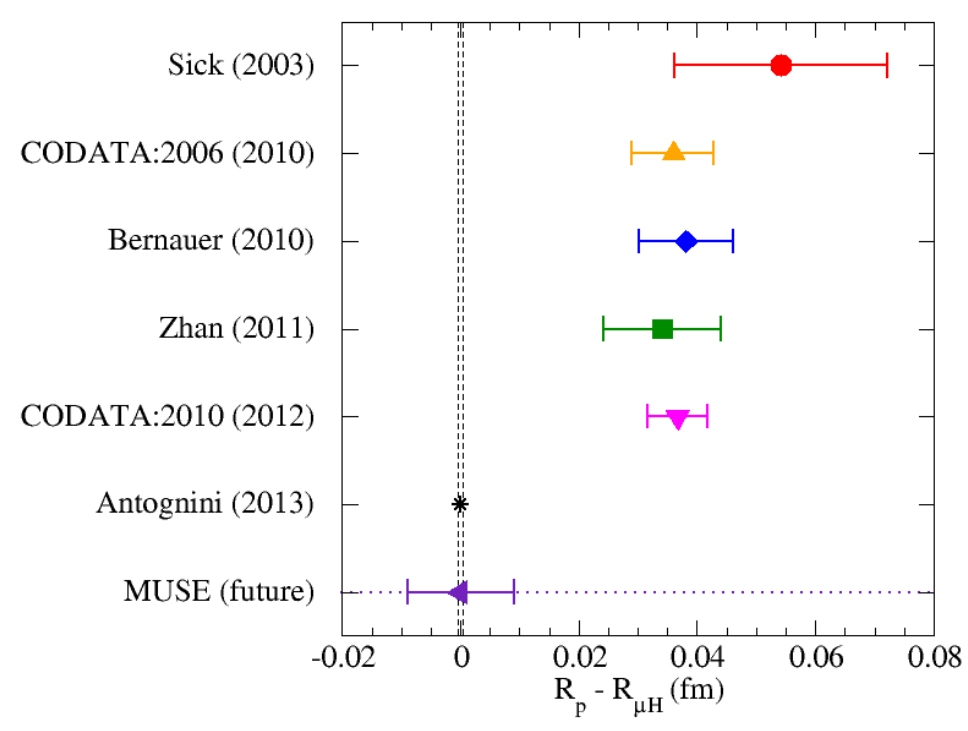

Figure 7: A conservative estimate of the sensitivity of MUSE to the current discrepancy of the proton radius in electron measurements relative to muonic hyodrgen. MUSE will measure this difference to similar sensitivity as current measurements; the point is arbitrarily placed at 0 .

\section{Acknowledgements}

R\&D work for the MUSE experiment is being funded by the National Science Foundation and the Department of Energy. This work is also supported in part by NSF PHY 1306126.

\section{References}

[1] R. Pohl et al., Nature 466 213-216, 2010.

[2] A. Antognini et al., Science 339 417-420, 2013.

[3] R. Pohl, R. Gilman, G.A. Miller, and K. Pachucki, Krzysztof, Ann. Rev. Nucl. Part. Sci. 63 175-204, 2013.

[4] I. Sick, Phys. Lett. B 576 62-67, 2003.

[5] J.C. Bernauer et al. [A1 Collaboration], Phys. Rev. Lett. 105 242001, 2010.

[6] X. Zhan et al., Phys. Lett. B 705 59-64, 2011.

[7] P.J. Mohr, B.N. Taylor, and D.B. Newell, Rev. Mod. Phys. 80 633-730, 2008.

[8] P.J. Mohr, B.N. Taylor, and D.B. Newell, Rev. Mod. Phys. 84 1527-1605, 2012.

[9] R.W. Ellsworth et al., Phys. Rev. 165 1449, 1968.

[10] I. Kostoulas et al., Phys. Rev. Lett. 32 489, 1974.

[11] R. Gilman et al. [MUSE Collaboration], arXiv:nucl-ex/1303.2160, 2013. 